\title{
EIGEN-PATCH IRIS SUPER-RESOLUTION FOR IRIS RECOGNITION IMPROVEMENT
}

\author{
Fernando Alonso-Fernandez*, Reuben A. Farrugia ${ }^{\dagger}$, Josef Bigun* \\ ${ }^{*}$ Halmstad University. Box 823. SE 301-18 Halmstad, Sweden. \\ $\dagger$ Department of CCE, University of Malta, Msida, Malta \\ Emails: feralo@hh.se, reuben.farrugia@um.edu.mt, josef.bigun@hh.se
}

\begin{abstract}
Low image resolution will be a predominant factor in iris recognition systems as they evolve towards more relaxed acquisition conditions. Here, we propose a super-resolution technique to enhance iris images based on Principal Component Analysis (PCA) Eigen-transformation of local image patches. Each patch is reconstructed separately, allowing better quality of enhanced images by preserving local information and reducing artifacts. We validate the system used a database of 1,872 near-infrared iris images. Results show the superiority of the presented approach over bilinear or bicubic interpolation, with the eigen-patch method being more resilient to image resolution reduction. We also perform recognition experiments with an iris matcher based 1D Log-Gabor, demonstrating that verification rates degrades more rapidly with bilinear or bicubic interpolation.
\end{abstract}

Index Terms - Iris hallucination, iris recognition, eigenpatch, super-resolution, Principal Component Analysis

\section{INTRODUCTION}

Iris has been shown as one of the most accurate biometric traits for human identification [1]. However, there is an increasing pressure towards relaxing acquisition conditions to allow acquisition 'at a distance' and 'on the move' [2]. This poses additional problems to the quality of acquired images, with the lack of pixel resolution the most evident. This paper addresses the problem of up-sampling, or increasing the size of a low-resolution image, which is due to for example a long acquisition distance to the object of interest. Low resolution is also an issue when bandwidth or storage limitations exist, such as in remote surveillance systems, personal devices or smartcards. In these cases, images are usually compressed e.g. via JPEG2000 [3], but image dimensions are kept constant. Nevertheless, resolution losses due to image compression are not within the scope of this paper.

Super-resolution (SR) techniques aim to reconstruct the missing high resolution (HR) image $\bar{Y}$ given a low resolution (LR) image $\bar{X}$. The LR image is modeled as the corresponding HR image manipulated by blurring $(B)$, warping $(W)$ and down-sampling $(D)$ as $\bar{X}=D B W \bar{Y}+\bar{n}(\bar{n}$ rep- resents additive noise). For simplicity, some works omit the warp matrix and noise, leading to $\bar{X}=D B \bar{Y}$. Two main SR approaches exist: reconstruction- and learning-based [4]. In reconstruction-based, sub-pixel shifts among multiple LR images are fused to obtain a HR image, therefore several LR images are needed as input. Alternatively, in learning-based approaches, the relationship between LR and HR images of a training database is modeled, and reconstruction can be done with only one LR image as input. Learning approaches generally outperform reconstruction methods and achieve higher magnification factors. Recently, SR techniques have been applied to biometric systems. Since Baker and Kanade first suggested SR algorithms specifically for faces [5], there has been a lot of research in face super-resolution (also called hallucination) [6]. Learning approaches have been also used with other biometrics such as iris [7-9]. However, one major limitation is that they try to develop a prototype iris using combination of complete images. Eigen-patches is a strategy which models a local patch using collocated patches from a dictionary, instead of using the whole image. Each patch is hallucinated separately, providing better quality reconstructed prototypes with better local detail and lower distortions.

This paper describes an iris super-resolution technique based on PCA hallucination of local patches inspired by the system of [10] for face images, referred to as Eigentransformation of local image patches. A PCA Eigentransformation is conducted in each image patch of the input LR image. The HR patch is then reconstructed as a linear combination of collocated HR patches of the training database. This way, every patch has its own optimal reconstruction coefficients, allowing to preserve local image information. Prior to the hallucination process, iris images are aligned with respect to the pupil center, since alignment is critical for the performance of SR systems. We use the CASIA-IrisV3-Interval database [11] of NIR iris images for our experiments. The presented hallucination system is superior to bilinear or bicubic interpolation, showing more resiliency to down-sampling. We also conduct verification experiments with a iris matcher based on 1D Log-Gabor wavelets, observing that recognition rates degrade more rapidly with bilinear or bicubic interpolation as resolution decreases. It is also shown that recognition performance is 
not degraded significantly until a down-sampling factor of $1 / 8$ or higher (this represents iris images of only $29 \times 29$ pixels). This allows for example to reduce the storage or data transmission requirements, two important requirements for biometric technologies to achieve massive adoption [2].

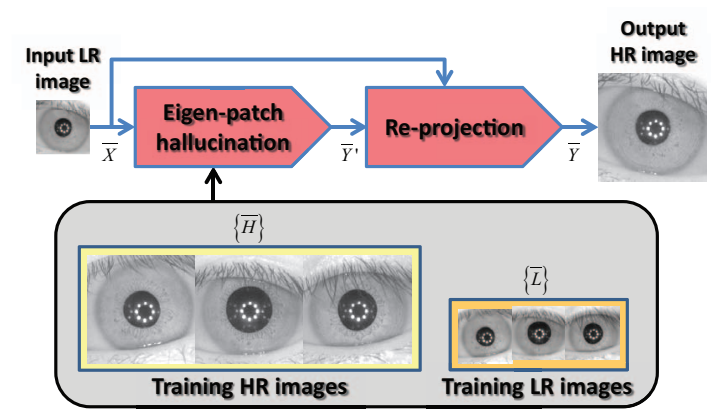

Fig. 1. Structure of the eigen-patch iris hallucination system.

\section{IRIS HALLUCINATION METHOD}

The structure of the hallucination method is shown in Figure 1. It is based on the eigen-patch hallucination method for face images of [10]. The system is described given next.

\subsection{Eigen-Patch Hallucination}

Given an input low resolution (LR) iris image $\bar{X}$, it is first separated into $N$ overlapping patches $\{\bar{x}\}=\left\{\overline{x_{1}}, \overline{x_{2}}, \cdots, \overline{x_{N}}\right\}$. Two super sets of basis patches are computed for each LR patch $\bar{x}_{i}$, separate from the input image $\bar{X}$, from collocated patches of a training database of high resolution images $\{\bar{H}\}$. One of the super sets, $\left\{\overline{h_{i}^{1}}, \overline{h_{i}^{2}}, \cdots, \overline{h_{i}^{M}}\right\}$, is obtained from collocated (HR) patches. By degradation (low-pass filtering and down-sampling) a low resolution database $\{\bar{L}\}$ is obtained from $\{\bar{H}\}$, and the other super set, $\left\{\overline{l_{i}^{1}}, \overline{l_{i}^{2}}, \cdots, \overline{l_{i}^{M}}\right\}$, is obtained similarly, but for $\{\bar{L}\} . M$ is the size (number of images) of the training set. A PCA Eigen-transformation is then conducted in each input LR patch $\bar{x}_{i}$ using the collocated patches $\left\{\overline{l_{i}^{1}}, \overline{l_{i}^{2}}, \cdots, \overline{l_{i}^{M}}\right\}$ of the LR facial training images to obtain the optimal reconstruction weights $\overline{c_{i}}=\left\{c_{i}^{1}, c_{i}^{2}, \cdots, c_{i}^{M}\right\}$ of each patch (see Figure 2). By allowing each LR patch of the input image to have its own optimal reconstruction weights, the HR patch will be closer to the input LR patch, therefore more local information can be preserved and less reconstruction artifacts appear. Once the reconstruction weights $\overline{c_{i}}$ of each patch are obtained, the HR patches are rendered using the collocated patches of the HR images of the training set $\{\bar{H}\}$. The reconstruction coefficients of the input image $\bar{X}$ using the LR patches is carried on to weight the HR basis set, which yields the preliminary reconstructed HR iris image $\bar{Y}^{\prime}$, after averaging the overlapping regions. Additional details of this Eigen-transformation procedure can be obtained in [10].

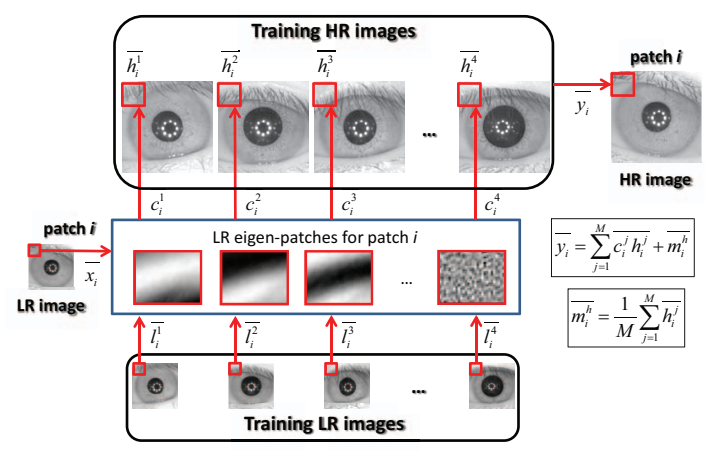

Fig. 2. Eigen-patch hallucination step.

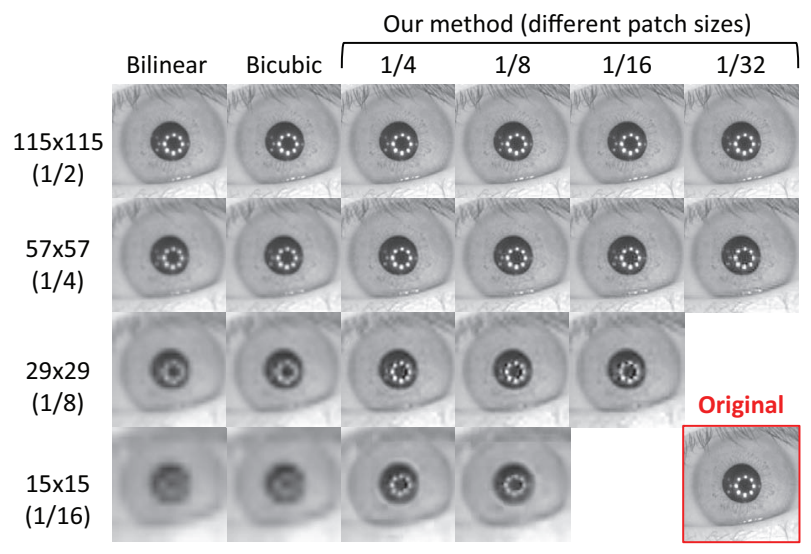

Fig. 3. Resulting HR hallucinated images for different downsampling factors and patch sizes (patch size is indicated in proportion to the size of the input LR image). The original HR image is also shown (bottom right).

\subsection{Image Re-projection}

A re-projection step is applied to $\bar{Y}^{\prime}$ to reduce artifacts and make the output image $\bar{Y}$ more similar to the input image $\bar{X}$. The image $\bar{Y}^{\prime}$ is re-projected to $\bar{X}$ via $\bar{Y}^{t+1}=\bar{Y}^{t}-$ $\tau U\left(B\left(D B \bar{Y}^{t}-\bar{X}\right)\right)$ where $U$ is the upsampling matrix. The process stops when $\left|\bar{Y}^{t+1}-\bar{Y}^{t}\right|$ is smaller than a threshold. We use $\tau=0.02$ and $10^{-5}$ as the difference threshold.

\section{IRIS MATCHER}

We conduct matching experiments of iris texture using 1D log-Gabor filters [12]. The iris region is unwrapped to a normalized rectangle of $20 \times 240$ pixels using the Daugman's rubber sheet model [13] and next, a 1D Log-Gabor wavelet is 


\begin{tabular}{|c|c|c|c|c|c|c|c|c|c|c|c|c|c|}
\hline \multirow{3}{*}{$\begin{array}{c}\text { LR size } \\
\text { (scaling) }\end{array}$} & & \multicolumn{6}{|c|}{ Full image } & \multicolumn{6}{|c|}{ Unwrapped iris region } \\
\hline & & \multirow[b]{2}{*}{ bilinear } & \multirow[b]{2}{*}{ bicubic } & \multicolumn{4}{|c|}{ Our method (patch size) } & \multirow[b]{2}{*}{ bilinear } & \multirow[b]{2}{*}{ bicubic } & \multicolumn{4}{|c|}{ Our method (patch size) } \\
\hline & & & & $1 / 4$ & $1 / 8$ & $1 / 16$ & $1 / 32$ & & & $1 / 4$ & $1 / 8$ & $1 / 16$ & $1 / 32$ \\
\hline \multirow{2}{*}{$\begin{array}{c}115 \times 115 \\
(1 / 2)\end{array}$} & psnr & 33 & 34.04 & 34.23 & 34.65 & 34.62 & 34.11 & 36.94 & 38.22 & 38.77 & 39.15 & 39.1 & 38.59 \\
\hline & ssim & 0.91 & 0.93 & 0.92 & 0.93 & 0.94 & 0.93 & 0.96 & 0.97 & 0.97 & 0.97 & 0.97 & 0.97 \\
\hline \multirow{2}{*}{$\begin{array}{c}57 \times 57 \\
(1 / 4)\end{array}$} & psnr & 28.36 & 29.18 & 29.91 & 29.9 & 29.53 & 28.78 & 31.64 & 32.35 & 32.69 & 32.72 & 32.38 & 31.83 \\
\hline & ssim & 0.79 & 0.8 & 0.8 & 0.81 & 0.8 & 0.78 & 0.85 & 0.87 & $\mathbf{0 . 8 8}$ & $\mathbf{0 . 8 8}$ & 0.88 & 0.86 \\
\hline \multirow{2}{*}{$\begin{array}{c}39 \times 39 \\
(1 / 6) \\
\end{array}$} & psnr & 26.21 & 26.8 & 27.98 & 28.05 & 27.86 & 27.61 & 29.61 & 30.21 & 30.71 & 30.85 & 30.73 & 30.64 \\
\hline & ssim & 0.73 & 0.74 & 0.74 & 0.75 & 0.75 & 0.75 & 0.79 & 0.81 & 0.81 & 0.82 & 0.82 & 0.82 \\
\hline \multirow{2}{*}{$\begin{array}{c}29 \times 29 \\
(1 / 8)\end{array}$} & psnr & 24.86 & 25.33 & 26.73 & 26.55 & 26.16 & - & 28.18 & 28.74 & 29.57 & 29.49 & 29.2 & - \\
\hline & ssim & 0.69 & 0.7 & 0.71 & 0.71 & 0.7 & - & 0.74 & 0.75 & $\mathbf{0 . 7 7}$ & 0.77 & 0.76 & - \\
\hline \multirow{2}{*}{$\begin{array}{c}23 \times 23 \\
(1 / 10) \\
\end{array}$} & psnr & 23.94 & 24.41 & 25.76 & 25.62 & 25.24 & - & 27.09 & 27.63 & 28.69 & 28.47 & 28.11 & - \\
\hline & ssim & 0.67 & 0.68 & 0.69 & 0.69 & 0.67 & - & 0.7 & 0.71 & $\mathbf{0 . 7 3}$ & 0.72 & 0.71 & - \\
\hline \multirow{2}{*}{$\begin{array}{l}19 \times 19 \\
(1 / 12) \\
\end{array}$} & psnr & 23.24 & 23.71 & 25.12 & 24.95 & 24.57 & - & 26.21 & 26.71 & 27.88 & 27.53 & 27.18 & - \\
\hline & ssim & 0.65 & 0.66 & 0.68 & 0.67 & 0.65 & - & 0.66 & 0.68 & 0.69 & 0.68 & 0.67 & - \\
\hline \multirow{2}{*}{$\begin{array}{c}17 \times 17 \\
(1 / 14) \\
\end{array}$} & psnr & 22.85 & 23.32 & 24.69 & 24.05 & - & - & 25.72 & 26.22 & 27.39 & 26.53 & - & - \\
\hline & ssim & 0.64 & 0.65 & 0.66 & 0.64 & - & - & 0.65 & 0.66 & 0.67 & 0.63 & - & - \\
\hline \multirow{2}{*}{$\begin{array}{l}15 \times 15 \\
(1 / 16) \\
\end{array}$} & psnr & 22.39 & 22.86 & 24.32 & 24.17 & - & - & 25.16 & 25.64 & 26.99 & 26.79 & - & - \\
\hline & ssim & 0.64 & 0.64 & 0.66 & 0.65 & - & - & 0.63 & 0.64 & 0.66 & 0.65 & - & - \\
\hline \multirow{2}{*}{$\begin{array}{l}13 \times 13 \\
(1 / 18)\end{array}$} & psnr & 21.8 & 22.25 & 23.94 & 23.69 & - & - & 24.43 & 24.87 & 26.36 & 26.22 & & \\
\hline & ssim & 0.63 & 0.63 & 0.65 & 0.65 & - & - & 0.6 & 0.62 & 0.63 & 0.63 & - & - \\
\hline
\end{tabular}

Table 1. Hallucination results with different down-sampling factors and different patch sizes (average values on the test dataset). Patch size is indicated in proportion to the size of the input LR image.

applied plus phase binary quantization to 4 levels. Matching between binary vectors is done using the normalized Hamming distance [13], which incorporates noise mask, so only significant bits are used. Rotation is accounted for by shifting the grid of the query image in counter- and clock-wise directions, and selecting the lowest distance, which corresponds to the best match between two templates. The iris region and corresponding noise mask is obtained by manual annotation of the database used (see next section).

\section{EXPERIMENTAL FRAMEWORK}

We use the CASIA Interval v3 iris database [11] for our experiments. This database has 2,655 NIR images of $280 \times 320$ pixels from 249 contributors captured in 2 sessions with a close-up iris camera, totalling 396 different eyes (the number of images per contributor and per session is not constant). Manual annotation of this database is available [14, 15], which has been used as input for our experiments. All images of the database are resized via bicubic interpolation to have the same sclera radius (we choose as target radius the average sclera radius $R=105$ of the whole database, given by the groundtruth). Then, images are aligned by extracting a square region of $231 \times 231$ around the pupil center (corresponding to about $1.1 \times R$ ). In case that such extraction is not possible (for example if the eye is close to an image side), the image is discarded. After this procedure, 1,872 images remain, which will be used for our experiments.

The dataset of aligned images has been divided into two sets, a training set comprised of images from the first 116 users ( $M=925$ images) used to train the eigen-patch hallucination method, and a test set comprised of the remaining 133 users (947 images) which is used for validation. We perform verification experiments with the iris matcher in the test set. We consider each eye as a different user. Genuine matches are obtained by comparing each image of a user to the remaining images of the same user, avoiding symmetric matches. Impostor matches are obtained by comparing the $1^{\text {st }}$ image of a user to the $2^{\text {nd }}$ image of the remaining users. With this procedure, we obtain 2,607 genuine and 19,537 impostor scores.

\section{RESULTS}

The 947 iris images of the test set are used as our high resolution (HR) reference images. We then down-sample these images via bicubic interpolation by a factor of $2 n$ (i.e. the image is resized to $1 /(2 n)$ of the original HR size), and the downsampled images are used as input LR images, from which hallucinated HR images are extracted. We also extract the normalized iris region (size $20 \times 240$ ) from both the hallucinated HR and the reference HR images, according to the algorithm of Section 3. The performance of the hallucination algorithm 
Scenario 1 (original vs. down-sampled)

\begin{tabular}{|c|}
\hline LR size (scaling) \\
\hline no downsampling \\
\hline $115 \times 115(1 / 2)$ \\
\hline $57 \times 57(1 / 4)$ \\
\hline $39 \times 39(1 / 6)$ \\
\hline $29 \times 29(1 / 8)$ \\
\hline $23 \times 23(1 / 10)$ \\
\hline $19 \times 19(1 / 12)$ \\
\hline $17 \times 17(1 / 14)$ \\
\hline $15 \times 15(1 / 16)$ \\
\hline $13 \times 13(1 / 18)$ \\
\hline
\end{tabular}

\begin{tabular}{|c|c|c|c|c|c|}
\cline { 3 - 7 } \multicolumn{2}{c}{} & \multicolumn{5}{c|}{ Our method (patch size) } \\
\hline bilinear & bicubic & $1 / 4$ & $1 / 8$ & $1 / 16$ & $1 / 32$ \\
\hline \multicolumn{7}{|c|}{0.76} \\
\hline $\mathbf{0 . 6 9}$ & $\mathbf{0 . 6 9}$ & 0.76 & 0.73 & 0.73 & 0.76 \\
\hline 0.69 & $\mathbf{0 . 6 8}$ & 0.8 & 0.73 & $\mathbf{0 . 6 8}$ & 0.77 \\
\hline 1.09 & 0.88 & 0.76 & 0.73 & 0.73 & $\mathbf{0 . 6 6}$ \\
\hline 1.61 & 1.42 & 1.11 & 1.15 & $\mathbf{1 . 0 8}$ & - \\
\hline 3.03 & 2.74 & $\mathbf{1 . 8 4}$ & 2.00 & 2.19 & - \\
\hline 4.96 & 4.48 & $\mathbf{3 . 4 0}$ & 3.86 & 4.33 & - \\
\hline 6.41 & 5.72 & $\mathbf{5 . 2 1}$ & 7.05 & - & - \\
\hline 10.39 & 9.59 & $\mathbf{7 . 2 9}$ & 7.72 & - & - \\
\hline 15.54 & 13.79 & 12.00 & $\mathbf{1 1 . 8 6}$ & - & - \\
\hline
\end{tabular}

Scenario 2 (down-sampled vs. down-sampled)

\begin{tabular}{|c|c|c|c|c|c|}
\cline { 3 - 7 } \multicolumn{2}{c|}{} & \multicolumn{5}{c|}{ Our method (patch size) } \\
\hline bilinear & bicubic & $1 / 4$ & $1 / 8$ & $1 / 16$ & $1 / 32$ \\
\hline \multicolumn{7}{|c|}{0.76} \\
\hline $\mathbf{0 . 6 1}$ & 0.73 & 0.73 & 0.72 & 0.76 & 0.76 \\
\hline 0.76 & $\mathbf{0 . 6 5}$ & 0.69 & 0.68 & 0.69 & 0.69 \\
\hline 1.07 & 0.83 & 0.76 & $\mathbf{0 . 7 0}$ & 0.92 & 0.83 \\
\hline 2.38 & 1.88 & $\mathbf{1 . 1 8}$ & 1.24 & 1.44 & - \\
\hline 5.25 & 4.41 & $\mathbf{1 . 8 9}$ & 2.20 & 2.82 & - \\
\hline 8.17 & 7.23 & $\mathbf{3 . 4 9}$ & 3.53 & 4.78 & - \\
\hline 9.86 & 9.41 & $\mathbf{4 . 2 9}$ & 6.47 & - & - \\
\hline 11.03 & 11.25 & $\mathbf{4 . 7 9}$ & 5.90 & - & - \\
\hline 12.40 & 12.23 & $\mathbf{6 . 4 4}$ & 6.94 & - & - \\
\hline
\end{tabular}

Table 2. Verification results (EER) of the two scenarios considered for different down-sampling factors and patch sizes (patch size is indicated in proportion to the size of the input LR image).
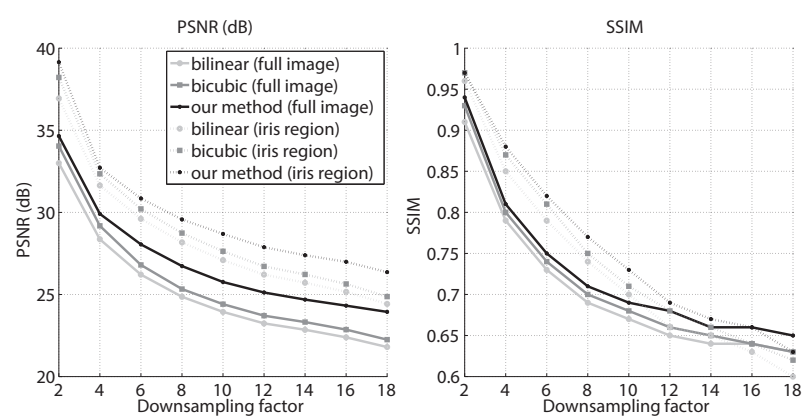

Fig. 4. Hallucination results with different down-sampling factors (the best performing patch size of each case according to Table 1 is selected).

is measured by computing the PSNR (in dBs) and SSIM values between the hallucinated HR image and the corresponding HR reference image. Results are shown in Table 1 . In the described hallucination method, size of LR patches is an important parameter. We therefore test different patch sizes, corresponding to $1 / 4,1 / 8,1 / 16$ and $1 / 32$ of the LR image size. Note that the exact size of the patch (in pixels) will depend on the size of the LR image. We define the patch size in proportion to the dimensions of the LR image to ensure that they cover the same relative size across different scaling factors. Overlapping between patches is $1 / 3$ of the patch size. We also compare our method with bicubic and bilinear interpolation. Figure 3 shows the hallucinated images (only for a selection of down-sampling factors for the sake of space).

It can be seen from Table 1 that the eigen-patch hallucination method outperforms the bilinear and bicubic interpolations, with the difference being higher for bigger downsampling factors. In other words, the eigen-patch method is more resilient as image resolution decreases. The latter can be better assessed in Figure 4 where we plot the results of Table 1 (the best performing patch size of each down-sampling factor, marked in bold, is selected). Although bilinear or bicu- bic interpolations have similar performance for small downsampling factors, the advantage of the utilized eigen-patch hallucination is more evident when the resolution of the LR image becomes very low. This can be also appreciated in the examples of Figure 3, where the blurring of bilinear and bicubic interpolations at very low resolutions is clearly evident.

With regards to the appropriate patch size, it can be observed in Table 1 that the best results are obtained with a big patch ( $1 / 4$ or $1 / 8$ of the LR image size, with $1 / 4$ being the best case at very low resolutions). A bigger patch allows a smoother reconstruction due to averaging a bigger region (see for example the case of $57 \times 57$ in Figure 3 , with more artifacts appearing for patch sizes of $1 / 16$ or $1 / 32$ ). It also has computational implications in terms of less patches to process per image, although patches have a bigger size.
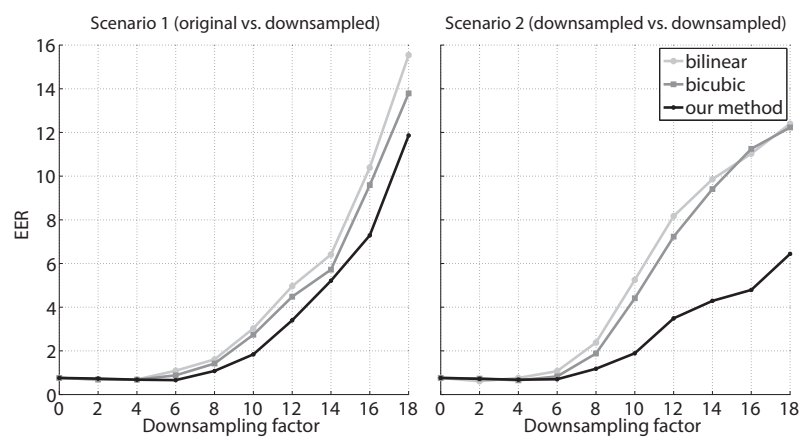

Fig. 5. Verification results (EER) of the two scenarios considered with different down-sampling factors (the best performing patch size of each case according to Table 2 is selected).

Next, we report verification experiments using hallucinated HR images. We consider two scenarios: 1) enrolment samples taken from original HR input images, and query samples from hallucinated HR images; and 2) both enrolment and query samples taken from hallucinated HR images. The first case simulates a controlled enrolment scenario with good 
quality images, while the second case simulates a totally uncontrolled scenario (albeit for simplicity, enrolment and query samples have similar resolution in our experiments). Results are given in Table 2 for different down-sampling factors and patch sizes. Similarly, the best cases (patch sizes) are plot in Figure 5. It can be observed that the verification performance using the proposed hallucination method is quite similar to bilinear and bicubic interpolations for small downsampling factors, but better performance is obtained with the hallucination method at very low resolutions. This is specially evident for the scenario 2, where the performance with bilinear or bicubic interpolations degrades rapidly, whereas the proposed method shows higher resiliency.

It is relevant that the performance of scenarios 1 and 2 with the proposed hallucination method is similar up to a down-sampling factor of $1 / 12$. In addition, performance is not affected until a down-sampling factor of 1/8 (image size of $29 \times 29$ ). This suggests that the size of both query and test images can be kept low without sacrificing performance, which is relevant for instance with devices having low storage capabilities or with low speed communication channels (recall that original HR images have a size of $231 \times 231$ ). In addition, from Figure 5 it can be observed that performance of scenario 2 is much better than scenario 1 at very low resolutions. When the size of input LR images is very low, the quality of hallucinated HR images is fairly different than the quality of original HR images (see Figure 3). As a result of such quality differences between enrolment and query images, it is better to match hallucinated HR images among themselves (scenario 2) than matching an hallucinated HR image against an original HR image (scenario 1). These results should not be taken as a general statement, since other iris matchers may lead to a different result. However, decreases in performance when matching biometric samples of heterogeneous quality have been also observed in other studies, e.g. [16].

\section{CONCLUSIONS}

As iris recognition systems evolve towards more relaxed acquisition conditions, variability in resolution will become more common [1]. In this paper, we propose the use of a iris super-resolution technique based on PCA Eigentransformation of local image patches (eigen-patches) [10] to increase the resolution of iris images. Experimental results on a database of near-infrared iris images show the superiority of the presented approach over bilinear or bicubic interpolation, with the eigen-patch method being more resilient to image resolution reduction. We also conduct iris matching experiments on the hallucinated images with a texture matcher based on 1D Log-Gabor wavelets. We consider two operational scenarios, one where original high-resolution images are matched against hallucinated high-resolution images (controlled enrolment), and another scenario where only hallucinated images are used (uncontrolled scenario). Ex- periments show that verification rates degrades more rapidly with bilinear or bicubic interpolation. It is also observed that recognition performance is not degraded significantly until image is down-sampled by $1 / 8$ or higher factors, allowing to use both query and test images of reduced size. The latter is of importance for example under low storage or data transmission capabilities. Future work includes separating left and right eye images to train the PCA algorithm with the aim of improving hallucination performance. The robustness of the hallucination method against training sets smaller than the one used here will also be studied, as well as the use of data acquired in visible range and under non-frontal and other perturbations. We will also explore the use of Manifold Learning approaches which model the non-linear relations between low and high resolution images.

\section{Acknowledgements}

This work was done while F. A.-F. was a visiting researcher at the University of Malta, funded by EU COST Action IC1106. Author F. A.-F. also thanks the Swedish Research Council for funding his research, and the CAISR program of the Swedish Knowledge Foundation.

\section{REFERENCES}

[1] K.W. Bowyer, K. Hollingsworth, P. J. Flynn, "Image understanding for iris biometrics: a survey," CVIU, vol. 110, pp. 281-307, 2007.

[2] A. K. Jain and A. Kumar, Second Generation Biometrics, chapter Biometrics of Next Generation: An Overview, Springer, 2010.

[3] G. W. Quinn, P. Grother, M. Ngan, "IREX IV: Part 2 - compression profiles for iris image compression," NISTIR 7978, 2014.

[4] S. C. Park, M. K. Park, M. G. Kang, "Super-resolution image reconstruction: a technical overview," Signal Proc Magaz, vol. 20(3), 2003.

[5] S. Baker, T. Kanade, "Limits on super-resolution and how to break them," IEEE TPAMI, vol. 24, no. 9, pp. 1167-1183, Sep 2002.

[6] N. Wang, D. Tao, X. Gao, X. Li, J. Li, "A comprehensive survey to face hallucination," IJCV, vol. 106, no. 1, pp. 9-30, 2014.

[7] K. Y. Shin et al., "Super-resolution method based on multiple multilayer perceptrons for iris recognition," Proc. ICUT, 2009.

[8] K. Nguyen, C. Fookes, S. Sridharan, S. Denman, "Quality-driven super-resolution for less constrained iris recognition at a distance and on the move," IEEE TIFS, vol. 6, no. 4, pp. 1248-1258, Dec 2011.

[9] K. Nguyen, S. Sridharan, S. Denman, C. Fookes, "Feature-domain super-resolution framework for gabor-based face and iris recognition," Proc. CVPR, 2012.

[10] H.-Y. Chen, S.-Y. Chien, "Eigen-patch: Position-patch based face hallucination using eigen transformation," in Proc. ICME, 2014.

[11] CASIA databases, "http://biometrics.idealtest.org/," .

[12] Libor Masek, "Recognition of human iris patterns for biometric identification," M.S. thesis, University of Western Australia, 2003.

[13] J. Daugman, "How iris recognition works," IEEE Trans. on Circuits and Systems for Video Technology, vol. 14, pp. 21-30, 2004

[14] F. Alonso-Fernandez, J. Bigun, "Near-infrared and visible-light periocular recognition with gabor features using frequency-adaptive automatic eye detection," IET Biometrics, 2015 (in press).

[15] H. Hofbauer, F. Alonso-Fernandez, P. Wild, J. Bigun, A. Uhl, “A ground truth for iris segmentation," Proc. ICPR, 2014

[16] F. Alonso-Fernandez, J. Fierrez, D. Ramos, J. Gonzalez-Rodriguez, "Quality-based conditional processing in multi-biometrics: Application to sensor interoperability," IEEE TSMC-A, vol. 40(6), 2010. 